\title{
Comparative transcriptome analysis of roots, stems and leaves of Isodon amethystoides reveals candidate genes involved in Wangzaozins biosynthesis
}

Fenglan Zhao ${ }^{1}$, Mengchu Sun ${ }^{1}$, Wanjun Zhang ${ }^{1}$, Chunli Jiang ${ }^{1}$, Jingtong Teng ${ }^{1}$, Wei Sheng ${ }^{1}$, Mingzhi Li ${ }^{2}$, Aimin Zhang ${ }^{1}$, Yongbo Duan ${ }^{1 *}$ and Jianping Xue ${ }^{1 *}$

\begin{abstract}
Background: Isodon amethystoides (Ben-th) Cy Wu et Hsuan is an important traditional medicinal plant endowed with pharmacological properties effective in the treatment of various diseases, including pulmonary tuberculosis. The tetracyclic diterpenoids, Wangzaozins (Wangzaozin A, glaucocalyxin A, glaucocalyxin B), are the major bioactive compounds of I. amethystoides. However, the molecular information about the biosynthesis of these compounds still remains unclear.

Results: An examination of the accumulated levels of Wangzaozins in I. amethystoides revealed considerable variations in the root, stem, and leaf tissues of this plant, indicating possible differences in metabolite biosynthesis and accumulation among various tissues. To better elucidate the tetracyclic diterpenoid biosynthesis pathway, we generated transcriptome sequences from the root, stem, and leaf tissues, and performed de novo sequence assembly, yielding 230,974 transcripts and 114,488 unigenes, with average N50 lengths of 1914 and 1241 bp, respectively. Putative functions could be assigned to 73,693 transcripts (31.9\%) based on BLAST searches against annotation databases, including GO, KEGG, Swiss-Prot, NR, and Pfam. Moreover, the candidate genes involving in the diterpenoid biosynthesis, such as CPS, KSL, were also analyzed. The expression profiles of eight transcripts, involving the tetracyclic diterpenoid biosynthesis, were validated in different I. amethystoides tissues by qRT-PCR, unraveling the gene expression profile of the pathway. The differential expressions of ISPD, ISPF and ISPH (MEP pathway), and laCPS and laKSL (diterpenoid pathway) candidate genes in leaves and roots, may contribute to the high accumulation of Wangzaozins in I. amethystoides leaves.
\end{abstract}

Conclusion: The genomic dataset and analyses reported here lay the foundations for further research on this important medicinal plant.

Keywords: Isodon amethystoides (ben-th) cy Wu et Hsuan, Transcriptome, de novo assembly, Tetracyclic diterpenoid, Wangzaozins

\footnotetext{
* Correspondence: yboduan@163.com; xuejp@163.com

${ }^{1}$ Key Laboratory of Resource Plant Biology of Anhui Province, College of Life

Sciences, Huaibei Normal University, Huaibei City, China

Full list of author information is available at the end of the article
}

(c) The Author(s). 2018 Open Access This article is distributed under the terms of the Creative Commons Attribution 4.0 International License (http://creativecommons.org/licenses/by/4.0/), which permits unrestricted use, distribution, and reproduction in any medium, provided you give appropriate credit to the original author(s) and the source, provide a link to the Creative Commons license, and indicate if changes were made. The Creative Commons Public Domain Dedication waiver (http://creativecommons.org/publicdomain/zero/1.0/) applies to the data made available in this article, unless otherwise stated. 


\section{Background}

The herbaceous genus Isodon (formerly Rabdosia), in the family Lemiaceae, contains a number of traditional medicinal plants that have a long history in traditional popular prescription in China. There are approximately 150 species of Isodon, nearly $70 \%$ of which are distributed in tropical and subtropical Asia [1, 2]. Many Isodon species have been shown to be effective in treating different illness, including I. japonicus for bleomycin-induced pulmonary fibrosis [3], I. eriocalyx for autoimmune encephalomyelitis amelioration [4], I. phyllostachys for its antiphlogistic and antibiotic actions [5], and I. rubescens for its anticancer, anti-inflammatory, and antibacterial properties [6]. Isodon amethystoides (Ben-th) Cy Wu et Hsuan, referred to as "Wang Zao Zi" and "Xiang Cha Cai" in China, is known for its broad applications as a folk remedy for abscesses, swollen sores, and tumors [7]. Unlike other traditional Chinese medicines needing concerted application, $I$. amethystoides is commonly used as a single drug for therapeutic purposes. Its ethanol extracts have shown a broad spectrum of antimicrobial efficacy, and in the 1980s were investigated as "Wang Zao Zi Soap" or "Wang Zao $\mathrm{Zi}$ Injection" for clinical trials in the Huaibei region of China [8]. The total healing rate reached $94 \%$ from 200 clinical cases with pneumonia, lung abscess, and pulmonary tuberculosis, thereby demonstrating its potential pharmacological value when used as a simple preparation.

Wangzaozins, including Wangzaozin A, glaucocalyxin A (Wangzaozin B), glaucocalyxin B (Wangzaozin C), are ent-kaurane-type tetracyclic diterpenoids isolated from Wangzaozi $[9,10]$. Having the same basic carbon skeleton, the three compounds differ in modified groups. Wangzaozin A has a hydroxyl on C-3 where it is a ketone in glaucocalyxin $\mathrm{A}$, and glaucocalyxin $\mathrm{B}$ is the acetylated structure of glaucocalyxin A [10]. Pharmacological studies have identified the bioactivities of Wangzaozins. For example, glaucocalyxin A has been widely studied for certain important biological activities, including antitumor [11], apoptosis induction [12, 13], antibacterial, anti-oxidative, anticoagulative, antithrombotic, antifibrotic, immune, and antineuroinflammatory activities [5, 14, 15]. Wangzaozin A, specifically isolated from Wang $\mathrm{Zao} \mathrm{Zi}$ and not from other Isodon species, has been proved to be effective in the treatment of pneumonia, lung abscess, and pulmonary tuberculosis when applied independently.

In plants, tetracyclic diterpenoids are synthesized via the plastid methylerythritol phosphate (MEP) pathway [16, 17]. The biosynthesis initiates with the reaction between glyceraldehyde 3-phosphate and pyruvate, which generates IPP and DMAPP. Both IPP and DMAPP can be converted into geranyl diphosphate (GPP), which generates geranylgeranyl-PP (GGPP) to further synthesize diterpenoids. GGPP is then converted to tetracyclic diterpenoids by ent-copalyl diphosphate synthase (CPS), and ent-kaurene synthase (KS) through sequential steps. For Isodon species, Li et al. [18] reported the role of two IeCPS genes in ent-copalyl diphosphate synthesis in I. eriocalyx via homologous cloning. Very recently, the diterpene synthases (diTPSs) including CPS and KSL have been identified in I. rubescens [19, 20].

DNA sequence information is a prerequisite for elucidating the molecular mechanism underlying diterpenoid biosynthesis. For non-sequenced medicinal plants, RNA-seq provides an efficient tool for us to generate large amount of omics data. For Isodon species, the transcriptomic data were reported in I. rubescens $[6,21]$, but it has not been recorded in I. amethystoides and other species yet. In the present study, we report transcriptome databases from the root, stem, and leaf tissues of one-year-old I. amethystoides plants. Through comprehensive analysis of the transcriptome data, we identified thousands of genes, including a set of putative genes involved in the diterpenoid biosynthesis pathway and a number of transcription factors (TFs). Moreover, we analyzed the transcripts involving tetracyclic diterpenoid Wangzaozins biosynthesis in different tissues. This work lays the foundations for functionally elucidating the genes involved in Wangzaozins biosynthesis, and molecular regulation of the biosynthesis of bioactive compounds in I. amethystoides.

\section{Results}

\section{Tetracyclic diterpenoid accumulation}

To examine the distribution pattern of tetracyclic diterpenoids, we determined the contents of three major bioactive tetracyclic diterpenoid compounds, Wangzaozin A, glaucocalyxin A, and glaucocalyxin B, in three tissues (root, leaf, and stem) of $I$. amethystoides. The three compounds have similar chemical structures, but differ in the structure of modified groups. The highest content of Wangzaozin A was observed in leaves, as high as $0.22 \%$ of DWT, followed by $0.031 \%$ in stems, which were 40 - and 6 -fold higher than that in roots, respectively. The highest accumulation of glaucocalyxin A was also found in leaves ( $0.16 \%$ of DWT), followed by stems, which were 48- and 7-fold higher than that in roots, respectively (Fig. 1). The content of glaucocalyxin $B$ in leaves was 6-fold higher than that in roots, whereas no glaucocalyxin B was detected in stems. Due to the distinct distributions of the major tetracyclic diterpenoids, all three tissues (root, stem, and leaf) were selected as experimental materials for studying the tetracyclic diterpenoid biosynthesis pathway via comparative transcriptome analysis.

\section{Illumina sequencing and read assembly}

To generate a transcriptome database for I. amethystoides, nine RNAseq libraries were constructed from the root, stem, and leaf tissues with three biological replicates. A total of $65.28 \mathrm{~Gb}$ clean data was recovered after trimming 


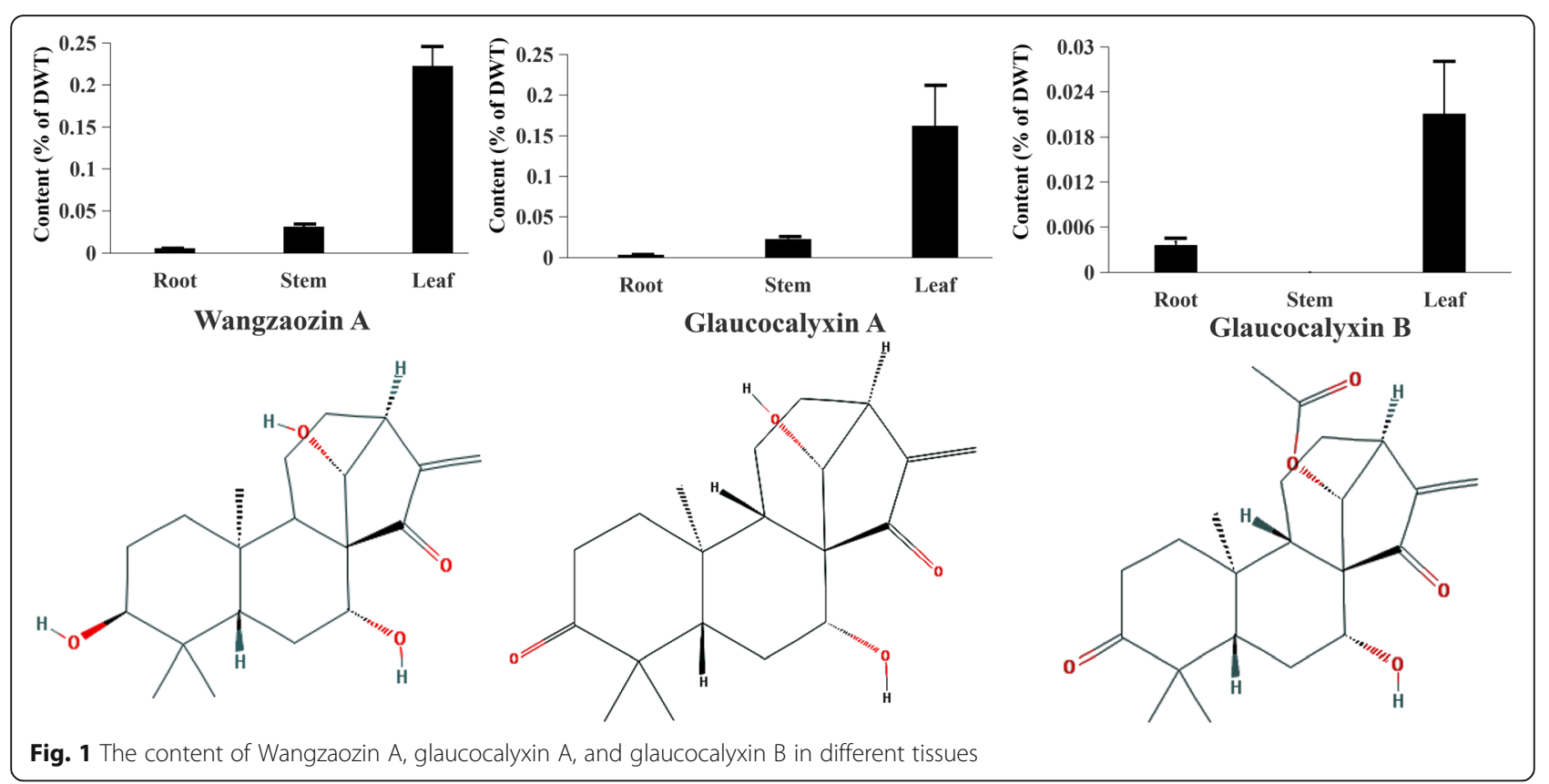

to remove adaptors, primer sequences, poly- $\mathrm{A}$ tails, and short and low-quality sequences. A range of 71 to 74 million for each tissue was obtained (Additional file 1: Table S2). Using the high-quality reads, 132,720 transcripts were assembled, with an average length of 1209 bp and N50 length of 1914 bp. Finally, 114,488 unigenes were identified. The total accumulated size of the assembled unigenes was approximately $98 \mathrm{Mb}$, with an N50 length of $1241 \mathrm{bp}$ and an average size of 858 bp. There were 42,851 (43.419\%) unigenes in the size range $300-500 \mathrm{bp}, 30,335$ (30.737\%) at 501-1000 bp, $14,572(14.765 \%)$ at $1000-$ $2000 \mathrm{bp}$, and 10,933 (11.078\%) > $2000 \mathrm{bp}$ (Additional file 2: Figure S1). The correlation indices between repeated samples were $\approx 0.9$ (Additional file 3: Table S3, Additional file 4: Figure S2), suggesting that the RNA-seq results are credible.

\section{Functional annotation}

The I. amethystoides transcripts were annotated by BLAST searches against several public databases. A statistical summary of these annotations is listed in Additional file 5: Table S4. Among the 114,488 unigenes, 25,101 (21.92\%) could be annotated in COG, 46557 (40.67\%) in GO, 17527 (15.31\%) in KEGG, 40517 (35.39\%) in KOG, 47026 (41.08\%) in Pfam, 47,100 (41.14\%) in Swiss-Prot, and 72,165 (63.03\%) in NR databases. Collectively, 73,693 transcripts $(64.37 \%)$ could be assigned at least one putative function from one of these databases. Functional annotations of the assembled transcripts with plant NRDB demonstrated that the majority of these are homologous to those in species including Sesamum indicum, Erythranthe guttata, and Vitis vinifera (Additional file 6: Figure S3). The remaining $35.63 \%$ of the transcripts had no significant protein matches in these databases. These non-matched transcripts may be novel or diverse proteins and long non-coding RNAs in I. amethystoides, or could be derived from less conserved $3^{\prime}$ or $5^{\prime}$ untranslated regions of the genes.

Various TF families, including the MYB, WRKY, and bHLH families, have been confirmed to be involved in secondary metabolite biosynthesis $[22,23]$. Transcriptome analysis of $I$. amethystoides revealed that 1373 unigenes (2\%) encode putative TFs that can be classified into $55 \mathrm{TF}$ families (Additional file 7: Table S5). Members of the $\mathrm{C} 2 \mathrm{H} 2 \mathrm{TF}$ family were the most abundant $(128,9.32 \%)$, followed by those in the ERF (113, 8.23\%), bHLH (101, 7.36\%), bZIP (97, 7.06\%), MYB-related (74, 5.39\%), and MYB (66, 4.81\%) families (Additional file 8: Figure S4). Identification of this large set of TFs, along with their expression profiling in individual tissues, provides a rich resource for further characterization of specific TFs in various biochemical pathways in I. amethystoides.

When GO was used to classify gene functions, 46,557 transcripts could be assigned to one or more GO terms within three domains and 51 functional categories (Additional file 9: Figure S5, Additional file 10: Table S6). Within the cellular component domain, the three most enriched categories were "cell part" (23,604, 50.70\%), "cell" $(23,485,50.44 \%)$, and "organelle" $(18,688,40.14 \%)$. In the molecular function domain, the three most matched categories were "catalytic activity" (25,576, 54.93\%), "binding" (22,564, 48.47\%), and "transporter activity" (3134, 6.73\%). In the biological process domain, the three most common categories were "metabolic process" (33,227, 45.09\%), 
"cellular process" (26,914, 36.52\%), and "single-organism process" (22,923, 31.11\%). The most commonly assigned functional categories in each domain were consistent with the results from other Isodon species [24, 25].

The assembled genes were assigned to the biological pathways described in KEGG to better understand the functions in specific metabolic pathways in I. amethystoides (Fig. 2). To systematically analyze inner cell metabolic pathways and complex biological behaviors, the annotated coding region sequences were mapped to the reference canonical pathways in the KEGG pathway database. As a consequence, 17,527 transcripts $(15.31 \%)$ could be assigned to five main categories: "metabolism," "genetic information processing," "environmental information processing," "cellular process," and "organismal systems," with 50 sub-categories and 124 pathways (Fig. 2, Additional file 11: Table S7). These enzymes have assigned functions in 23 secondary metabolic pathways in KEGG (Table 1). Among these, 230 transcripts encode key enzymes involved in the pathways for tetracyclic diterpenoid biosynthesis, including the synthesis of the tetracyclic diterpenoid backbone (134 transcripts), monoterpenoids (2 transcripts), diterpenoids (29 transcripts), sesquiterpenoid (1 transcript), and other terpenoids (66 transcripts). Nine transcripts with high homology with GA oxidase sequences. Two hundred and eighty-eight transcripts were related to genes involved in the flavonoid biosynthesis pathway, including the phenylpropanoid (205 transcripts), flavonoid (64 transcripts), and flavone and flavonol (19 transcripts) biosynthesis pathways. Ninety transcripts were associated with alkaloid biosynthesis, including indole alkaloid biosynthesis (4 transcripts), isoquinoline alkaloid biosynthesis (38 transcripts), and tropane, piperidine, and pyridine alkaloid biosynthesis (48 transcripts). Identification and future characterization of transcripts involved in these different metabolic pathways will help us to better understand their functions in the biosynthesis of active compounds in Isodon plants.

\section{Differential gene expression analysis}

To investigate the differential gene expression among different tissues, the high-quality reads from individual samples were mapped onto the I. amethystoides transcriptome using CLC Genomics Workbench (v8.0.2). DEGs were identified (Additional file 12: Table S8) using the DESeq package with FDR $\leq 0.01$ and at least a 2-fold expression change among different tissues [26]. The number of DEGs was highest between the root and leaf, and lowest between the stem and leaf. Furthermore, the DEGs in one tissue with respect to those in the other two tissues were also investigated. Roots contained the largest number of most highly expressed transcripts, with 8906 transcripts being more abundant than in the

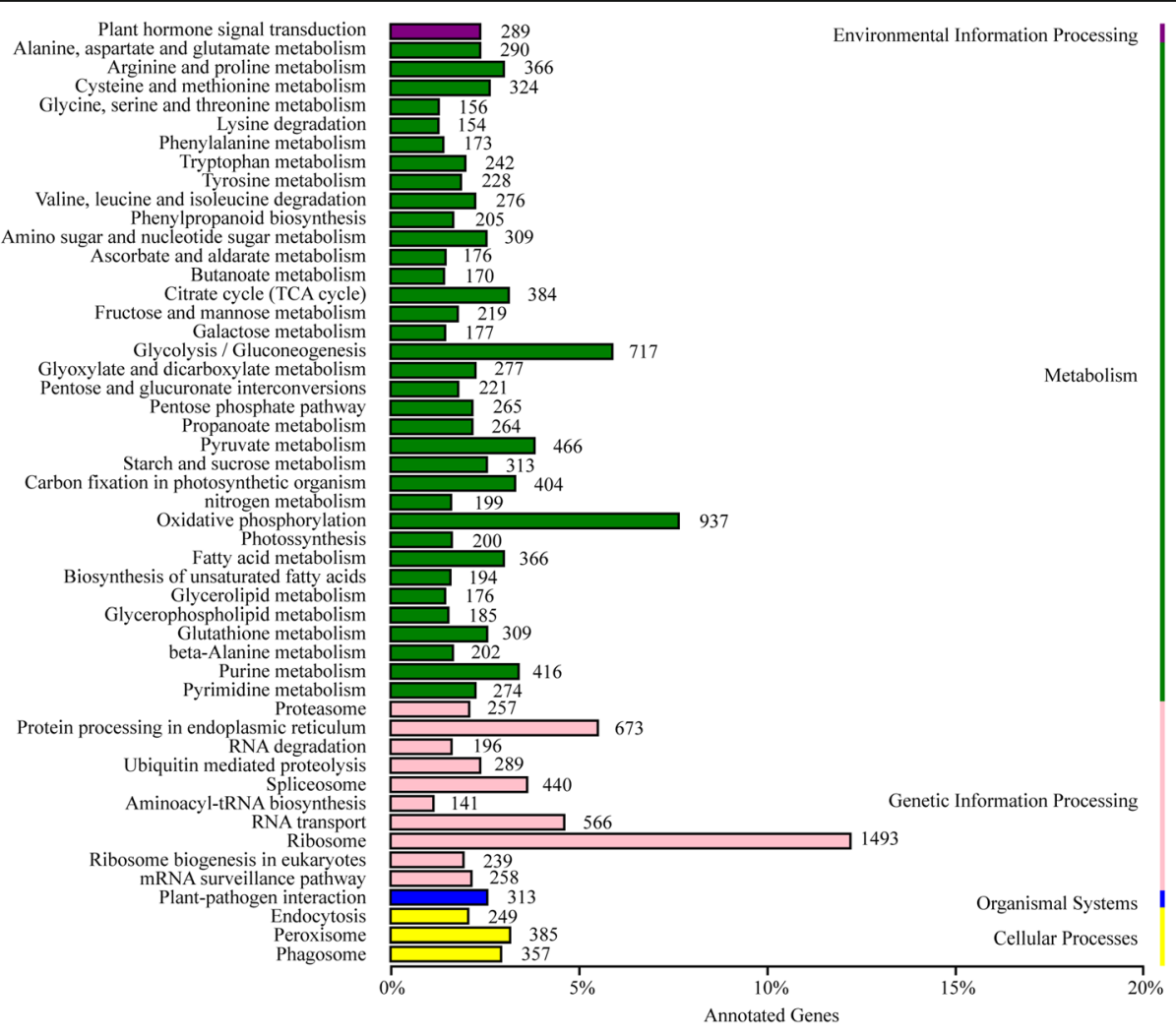

Fig. 2 KEGG pathway classification map. Genes were divided into five branches according to corresponding biological pathways 
Table 1 Secondary metabolism pathways in Isodon amethystoides

\begin{tabular}{|c|c|c|c|}
\hline Pathway ID & Pathways & $\begin{array}{l}\text { Functional } \\
\text { categories }\end{array}$ & $\begin{array}{l}\text { Unique gene } \\
\text { number }\end{array}$ \\
\hline ko00100 & Steroid biosynthesis & 21 & 74 \\
\hline ko00130 & $\begin{array}{l}\text { Ubiquinone and other } \\
\text { terpenoid-quinone } \\
\text { biosynthesis }\end{array}$ & 17 & 66 \\
\hline ko00230 & Purine metabolism & 91 & 416 \\
\hline ko00232 & Caffeine metabolism & 9 & 11 \\
\hline ko00400 & $\begin{array}{l}\text { Phenylalanine, tyrosine, } \\
\text { and tryptophan } \\
\text { biosynthesis }\end{array}$ & 36 & 89 \\
\hline ko00860 & $\begin{array}{l}\text { Porphyrin and chlorophyll } \\
\text { metabolism }\end{array}$ & 32 & 130 \\
\hline ko00900 & $\begin{array}{l}\text { Terpenoid backbone } \\
\text { biosynthesis }\end{array}$ & 28 & 134 \\
\hline ko00901 & Indole alkaloid biosynthesis & 4 & 4 \\
\hline ko00902 & Monoterpenoid biosynthesis & 2 & 2 \\
\hline ko00903 & $\begin{array}{l}\text { Limonene and pinene } \\
\text { degradation }\end{array}$ & 6 & 94 \\
\hline ko00904 & Diterpenoid biosynthesis & 8 & 38 \\
\hline ko00905 & Brassinosteroid biosynthesis & 6 & 6 \\
\hline ko00906 & Carotenoid biosynthesis & 24 & 78 \\
\hline ko00908 & Zeatin biosynthesis & 7 & 46 \\
\hline ko00909 & Sesquiterpenoid biosynthesis & 1 & 1 \\
\hline ko00940 & Phenylpropanoid biosynthesis & 23 & 205 \\
\hline ko00941 & Flavonoid biosynthesis & 16 & 64 \\
\hline ko00942 & Anthocyanin biosynthesis & 1 & 1 \\
\hline ko00944 & $\begin{array}{l}\text { Flavone and flavonol } \\
\text { biosynthesis }\end{array}$ & 7 & 19 \\
\hline ko00945 & $\begin{array}{l}\text { Stilbenoid, diarylheptanoid, } \\
\text { and gingerol biosynthesis }\end{array}$ & 6 & 49 \\
\hline ko00950 & $\begin{array}{l}\text { Isoquinoline alkaloid } \\
\text { biosynthesis }\end{array}$ & 8 & 38 \\
\hline ko00960 & $\begin{array}{l}\text { Tropane, piperidine, and } \\
\text { pyridine alkaloid biosynthesis }\end{array}$ & 9 & 48 \\
\hline ko00965 & Betalain biosynthesis & 1 & 1 \\
\hline
\end{tabular}

other organs, while 6410 were less abundant (Fig. 3a). In contrast, in stems, 5072 genes were expressed at a higher level than in roots and leaves, while 7792 genes were expressed at a lower level. In addition, a total of 6354 transcripts exhibited tissue-specific expression, with 1127, 760, and 4467 transcripts being specifically expressed in root, stem, and leaf, respectively (Fig. 3b). Among the identified DEGs, 1373 encoded TFs, some of which were specifically expressed in the root, stem, and leaf (Additional file 12: Table S8). Future characterization of specific TFs is required for a better understanding of the gene expression profiles and regulation in the various biochemical pathways in I. amethystoides.
To explore the possible genes regulating the distinct distribution of Wanzaozins, we further performed KEGG enrichment analyses with the DEGs among root, leaf, and stem. These DEGs significantly enriched in a number of pathways. Between root and leaf, photosynthesis, plant hormone signal transduction, zeatin biosynthesis, carotenoid biosynthesis, photosynthesis - antenna proteins, diterpenoid biosynthesis, porphyrin and chlorophyll metabolism, and phenylpropanoid biosynthesis, are the first eight pathways showing the greatest differential expression (Fig. 4a). Between leaf and stem, the eight pathways showing significant enrichment were photosynthesis, plant hormone signal transduction, phenylpropanoid biosynthesis, starch and sucrose metabolism, carotenoid biosynthesis, cyanoamino acid metabolism, $\mathrm{ABC}$ transporters, and phenylalanine metabolism (Fig. 4b). Between root and stem, photosynthesis, photosynthesis - antenna proteins, phenylalanine metabolism, phenylpropanoid biosynthesis, steroid biosynthesis, carotenoid biosynthesis, diterpenoid biosynthesis were the eight categories showing significant enrichment (Fig. 4c). The pathways of primary metabolism, including photosynthesis, amino acid metabolism, and starch and sucrose metabolism, which are essential to plant growth, were common in all three tissues. With the exception these common pathways, the enriched secondary metabolic pathways, including diterpenoid and flavonoid biosynthesis, were also detected between different tissues, particularly between roots and above-ground parts (Additional file 13: Table S9), indicating the possible distinct distribution of secondary metabolites among various tissues.

\section{Putative genes involved in the tetracyclic diterpenoid biosynthesis}

Totally 29 transcripts classified into 12 enzyme categories were identified by using transcriptome data to examine the KEGG database (Additional file 14: Table S10). NCBI blast showed that these candidate transcripts displayed 67-99\% homology with their respective best matches.

The putative pathway of tetracyclic diterpenoids Wangzaozins biosynthesis was proposed (Fig. 5). In the MEP pathway, 14 transcripts were identified, including those of four 1-deoxy-D-xylulose-5-phosphatesynthases (DXS) and two 1-deoxy-D-xylulose-5-phosphate reductoisomerases (DXR). DXS and DXR promote the generation of 2-C-methyl-derythritol 4-phosphate (MEP) from the substrate D-glyceraldehyde 3-phosphate and pyruvate. Of the four DXS genes, IaDXS1 expressed similarly in all three tissues, whereas IaDXS4 showed the highest expression in stem. Both IaDXR1 and IaDXR2 displayed a constitutive expression in three tissues, and IaDXR1 had a remarkably higher expression than IaDXR2. These suggest that IaDXS1, IaDXS4, and IaDXR1 are important for the generation of MEP and 


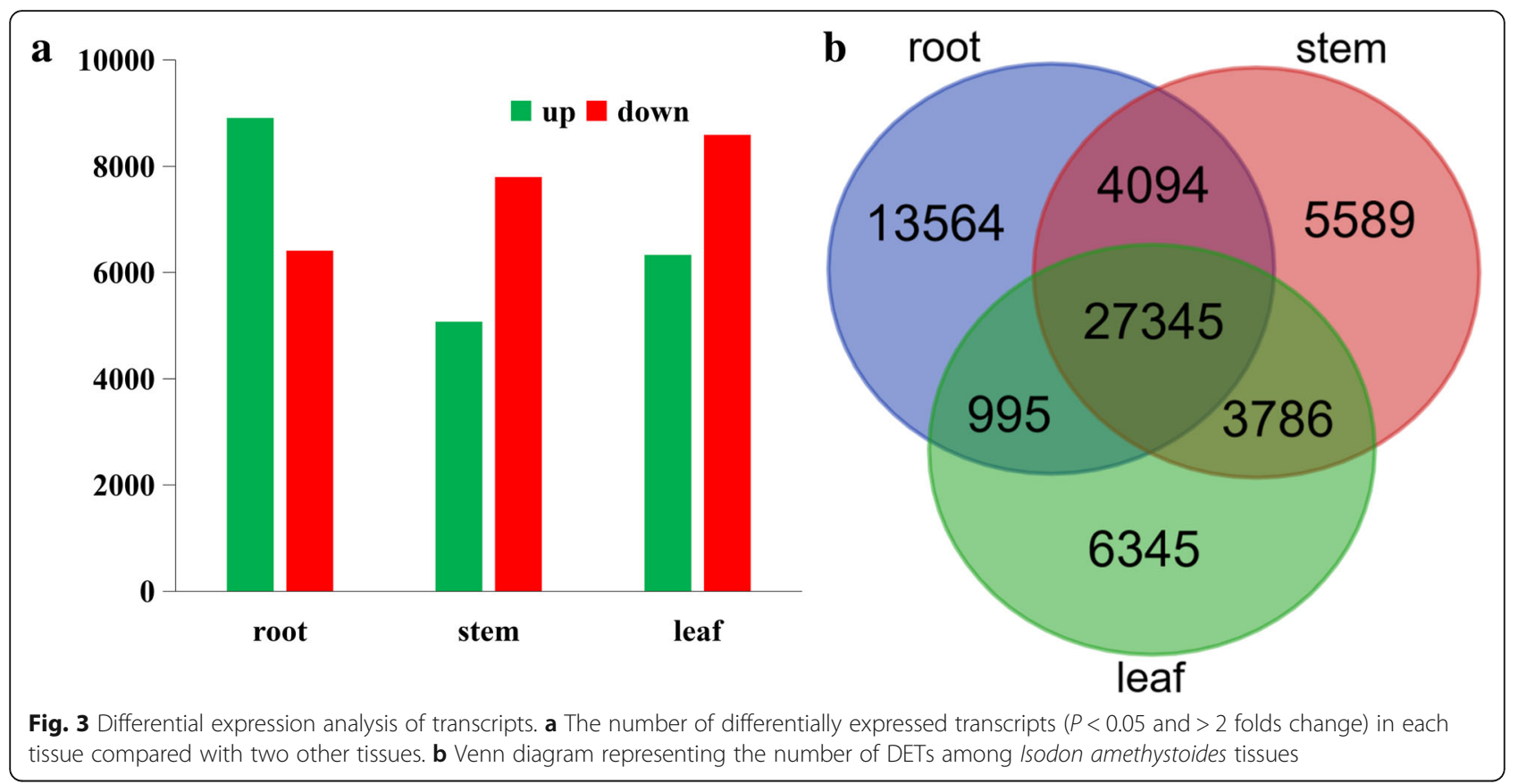

deserve further functional characterization. MEP further reacts under the catalysis of following enzymes. In our transcriptomic dataset, only one transcript for 2-C-methylD-erythritol 4-phosphate cytidylyltransferase (ISPD), 1-hydroxy-2-methyl-2-(E)-butenyl 4-diphosphate reductase (ISPF), and 1-hydroxy-2-methyl-2-(E)-butenyl 4-diphosphate reductase (ISPH) each was identified, and their expressions were 2 to 3 - fold higher in leaf than root, inferring that these three genes may be single copy genes. There are two transcripts for both IaISPE and IaISPG, which displayed constitutive expressions or predominant expression in one of the three tissues. These genes may lead to high efficiency of tetracyclic diterpenoid substrate biosynthesis in leaf of $I$. amethystoides.

The tetracyclic diterpenoid biosynthesis involves geranyl diphosphate synthase (GPPS), geranylgeranyl diphosphate synthase (GGPPS), ent-copalyl diphosphate synthase (CPS) and ent-kaurene synthase (KSL). In total, 15 transcripts were found to be involved in this pathway. Two GPPS and four GGPPS transcripts were identified in our dataset. IaGPPS1 was expressed predominantly in root and rarely detectable in leaf, while IaGPPS 2 showed the highest expression in stem. IaGGPS1 was expressed at a considerably higher level in above ground parts than roots, whereas IaGGPS2 showed a similar expression among three tissues.

With regard to CPS, an important enzyme in the diterpenoid pathway, 5 transcripts were found in I. amethystoides. IaCPS4 showed constitutive expression among three tissues, while IaCPS1 had a higher expression in stem than other tissues. In the dataset we found one CPS transcript, IaCPS3 (DN34471_c0_g1), containing

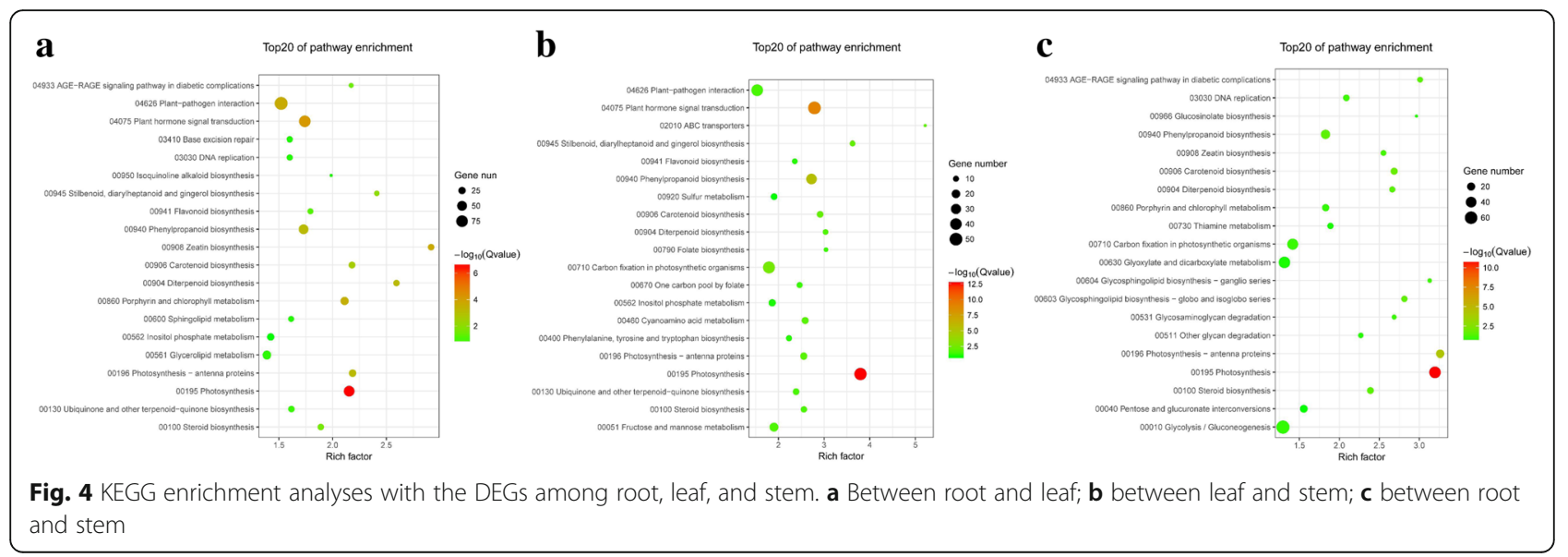




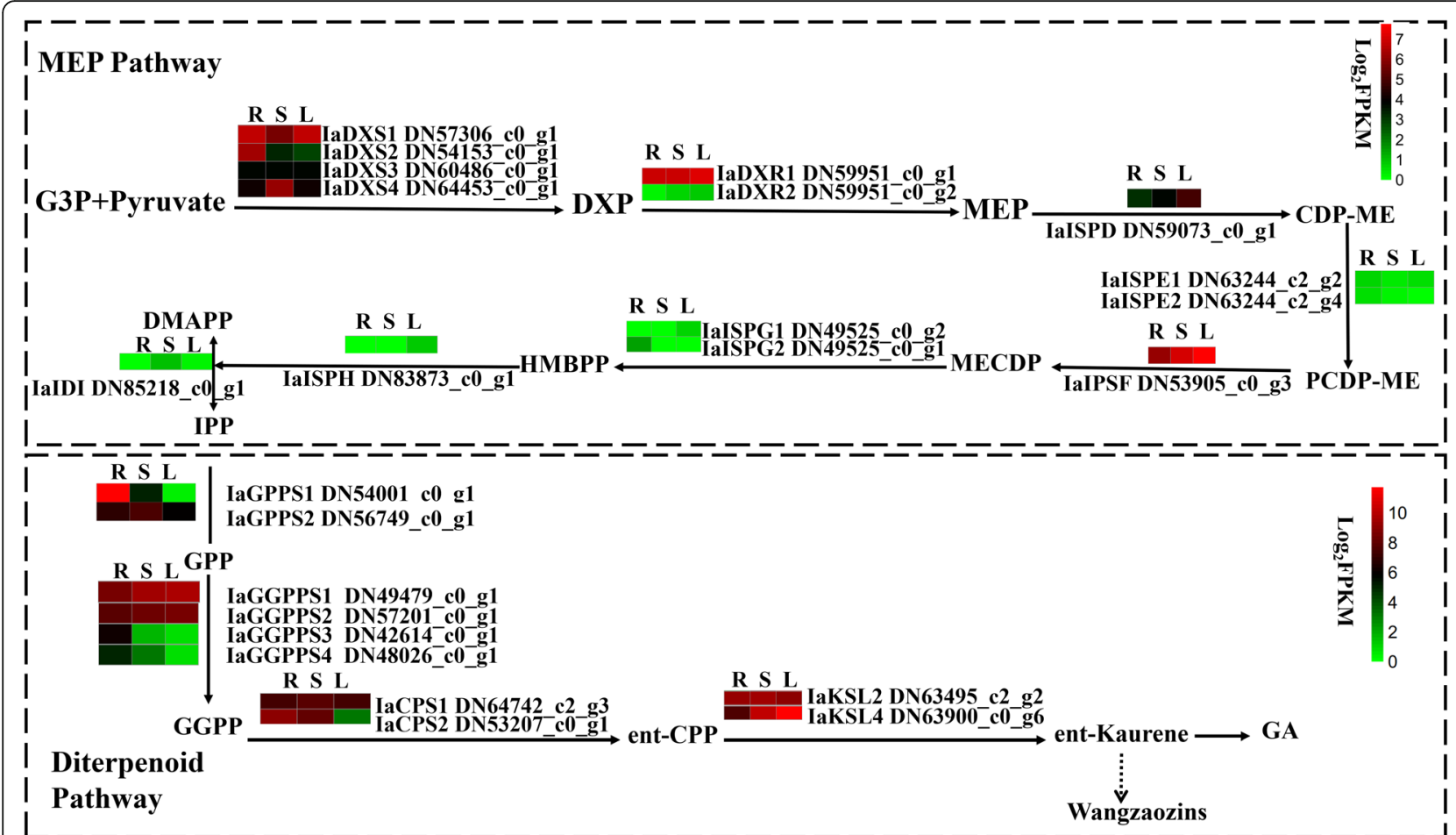

Fig. 5 Schematic representation of the proposed diterpenoid biosynthesis pathway and differential expression of genes identified. The transcriptomic data $\left(\log _{2}\right.$ FPKM) for each DEG represent the expression in root (R), stem $(S)$, and leaf $(L)$ on heat map from the left

the complete open reading frame, was expressed predominantly in leaves. Phylogenetic analysis indicated that IaCPS1 fell into a same clade with IeCPS2, and it also has a high similarity with IrCPS4 (Fig. 6). IeCPS2 and IrCPS4 have been determined to be involved in pharmacologically active Isodon diterpenoids biosynthesis $[18,19]$. Therefore, the CPS candidate genes, especially IaCPS1 (DN64742_c2_g3), may have important roles in Wangzaozins biosynthesis.

For ent-kaurene synthase, we detected 4 possible transcripts. Except for IaKSL2, which showed a similar expression level in all three tissues, the other three were expressed at a considerably higher level in leaves and stems. Particularly, IaKSL4 was highly expressed in leaf and stem. These KLS candidate transcripts shared high homologies with known counterparts in other species (Fig. 6). IaKSL2 and IaKSL4 were closely related to IrKSL5 and IrKSL4, respectively. IrKSL2 and IrKSL5 have been shown to react with ent-CPP [19]. The results indicate that KSL genes (IaKSL2 and IaKSL4) having a high or predominant expression, may be a cause of accumulation of Wangzaozins in $I$. amethystoides leaves.

The transcriptomic data for different tissues would be helpful for obtaining the CDS of putative genes involved in Wangzaozins biosynthesis pathways and unraveling their family members and functions in I. amethystoides.
Expression patterns of gibberellin (GA) biosynthesis candidate genes among different tissues

Wangzaozins, belonging to ent-kaurane-type tetracyclic diterpenoids $[9,10]$, share the same requisite skeletal structure with gibberellin, as well as oridonin. Oridonin has been reported to have the same precursor of ent-kaurene with gibberellin [19, 27]. Therefore, ent-kaurene is thought to be the common substrate for biosynthesis of gibberellin and Wangzaozins. Because plant hormone signal transduction showed significant enrichment between root and leaf, and stem and leaf, we analyzed the expressions of GA biosynthesis genes among three tissues. Nine transcripts that are potentially involved in gibberellin biosynthesis were detected: three encoding gibberellin 20-oxidases (GA20ox), two gibberellin 3-oxidases (GA3ox) two ent-kaurene oxidases (KO) and two ent-kaurenoic acid oxidases (KAO) (Fig. 7a). Except for IaGA3ox1 being expressed consistently, all other genes showed differential expression patterns among three tissues. Phylogenetic analysis showed that these GA biosynthesis related candidate genes had a close genetic relationship with those reported in other Isodon species or dicots (Fig. 7b). The differential expressions of these candidate transcripts may lead to the difference in GA production among different tissues. 

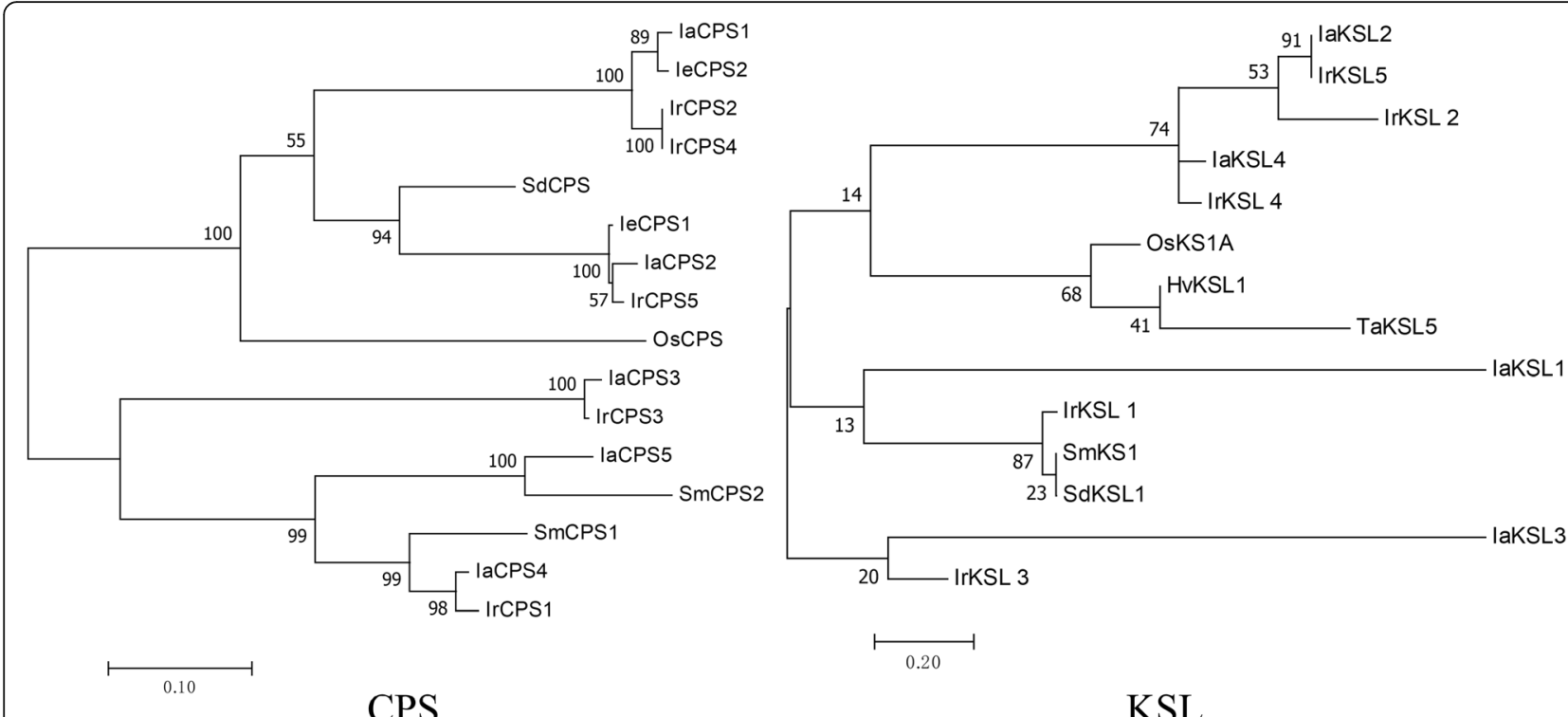

CPS

KSL

Fig. 6 Phylogenetic analysis of Isodon amethystoides CPS and KSL candidate genes by the maximum likelihood method with MEGA software. I. amethystoides CPS and KSL transcripts listed in Additional file 14: Table S10 were blasted in NCBI to obtain the CDS information for phylogenetic analysis. leCPS1, I. eriocalyx ent-copalyldiphosphate synthase (AEP03177); leCPS2, I. eriocalyx ent-copalyldiphosphate synthase (AEP03175); IrCPS1, I. rubescens ent-copalyldiphosphate synthase (KU180499); IrCPS3, I. rubescens copalyl diphosphate synthase 3 (APJ36373); IrCPS4, I. rubescens copalyl diphosphate synthase 4 (APJ36374); IrCPS5, I. rubescens copalyl diphosphate synthase 5 (APJ36375); SmCPS1, Salvia miltiorrhiza copalyl diphosphate synthase 1 (KC814639); SmCPS2, S. miltiorrhiza copalyl diphosphate synthase 2 (AHJ59322); SdCPS, Scoparia dulcis entcopalyldiphosphate synthase (BAD91286); OsCPS, Oryza sativa ent-copalyldiphosphate synthase (BAD424492); IrKSL1, I. rubescens ent-kaurene synthase (KUI180504); IrKSL2, I. rubescens ent-kaurene synthase (KUI180505); IrKSL3, I. rubescens ent-kaurene synthase (KUI180506); IrKSL4, I. rubescens ent-kaurene synthase (KX580633); IrKSL5, I. rubescens ent-kaurene synthase (KX580635); TaKSL5, Triticum aestivum nerolidol synthase (BAL41692); OsKSL1, Oryza sativa ent-kaurene synthase (AAQ72559); HVKSL1, Hordeum vulgare ent-kaurene synthase (AAT49066). Numbers on branches indicate the bootstrap percentage values calculated from 1000 bootstrap replicates

\section{qRT-PCR validation of candidate genes involved in Wangzaozins biosynthesis}

To validate the transcriptome analysis data and also to evaluate the differential expression profile among different tissues, we selected eight transcripts cognate to Wangzaozins biosynthesis for qRT-PCR analysis. The expressions of these transcripts based on FPKM value are shown in Fig. 8. After validation, it was found that all eight transcripts showed a similar expression pattern based on FPKM value, though there are some differences in expression folds. Both IaDXS1 and IaDXR1 showed a similar expression among three tissues. The single copy transcripts IaISPD, IaISPF and IaISPH had a predominant expression in leaf or stem. Interestingly, IaISPH was not detected in root by qRT-PCR. IaGGPPS2 and IaCPS1 showed an approximately 2-fold higher expression in stems than root. IaKSL4 was expressed predominantly in leaf, with a 159-fold increased expression compared to that in the root respectively. The qRT-PCR results of all eight genes were in a good agreement with the transcriptomic data. The results further confirm the high reliability of transcriptomic data from three replicates, which will be helpful for understanding the biosynthesis pathway of Wangzaozins in I. amethystoides.

\section{Discussion}

Tetracyclic diterpenoids are a kind of 20-carbon $\left(\mathrm{C}_{20}\right)$ isoprenoids that are endowed with broad-spectrum bioactivities. Their biosynthesis and accumulation are usually restricted to specialized tissues or cell types. Usually the specialized diterpenoids are stored in the place of their synthesis [28]. Therefore, the tissue-specific accumulations of tetracyclic diterpenoids are associated with differential gene expression rather than their translocation to specialized tissues [16].

For non-model species lacking a well-studied genetic background, de novo transcription analysis provides an effective means of generating comprehensive information for the discovery of novel genes, and construction of gene expression networks for specific tissues [29-31]. In this study, a total of $65.28 \mathrm{~Gb}$ clean data were obtained from nine RNA-seq libraries of root, stem, and leaf. De novo assembly generated 114,488 transcripts with a total accumulated size of $\approx 98 \mathrm{Mb}$. The assembled data had an average N50 length of $1241 \mathrm{bp}$, which is similar to that recorded previously in other non-model plants, such as Andrographis paniculata [32, 33] and Raphanus sativus [34]. 


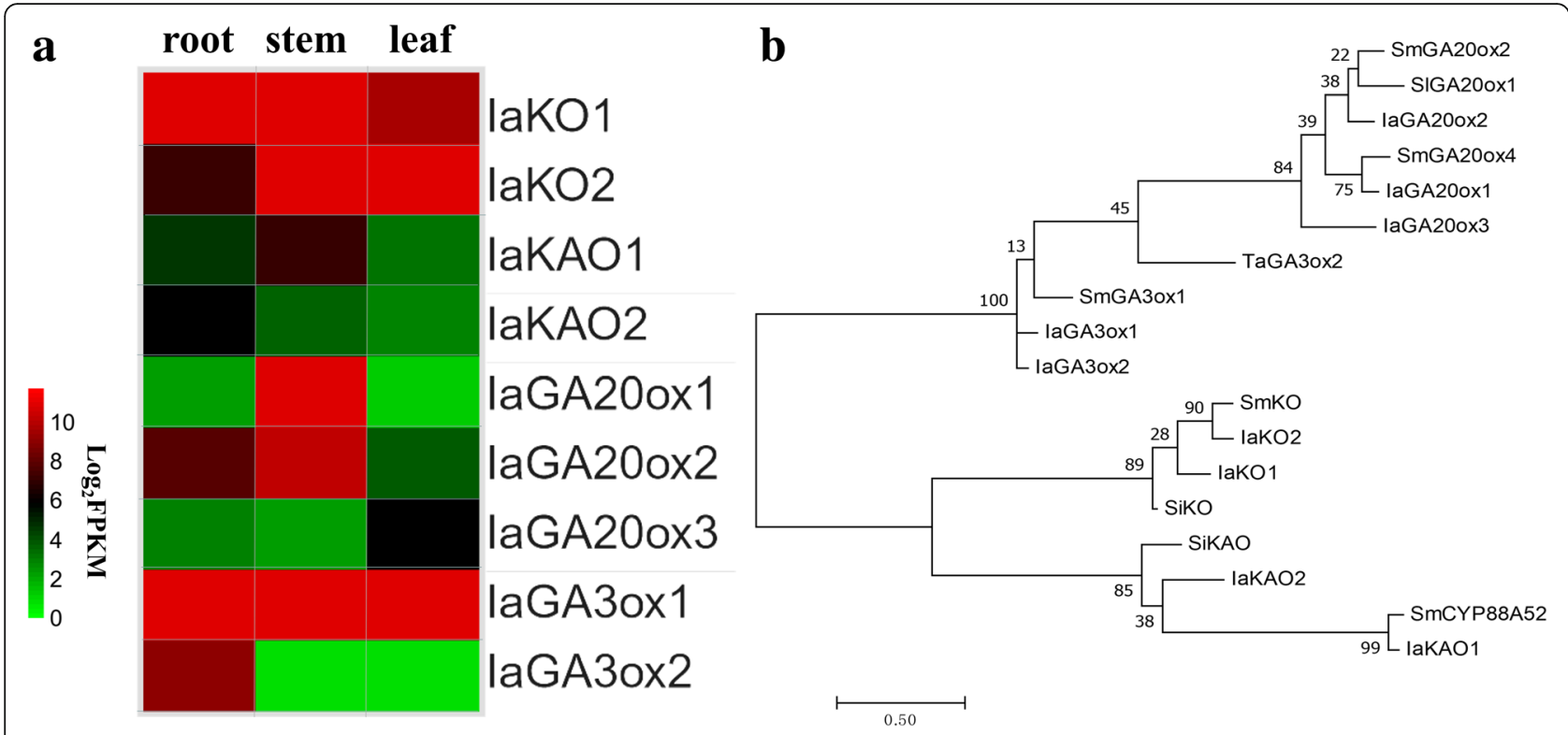

Fig. 7 Expression of candidate genes associated with gibberellins biosynthesis. a Schematic representation of gibberellin biosynthesis associated transcripts by heatmap based on FPKM values; $\mathbf{b}$ phylogenetic analysis of potential genes by the maximum likelihood method with MEGA software. laKO1, DN62518_c0_g1; laKO2, DN63069_c0_g1; laKAO1, DN47083_c0_g1; laKAO2, DN60719_c0_g6; laGA20ox1, DN51838_c0_g1; laGA20ox2, DN59747_c0_g2; laGA20ox3, DN51642_c0_g1; laGA3ox1, DN58138_c0_g2; laGA3ox2, DN58138_c0_g1; SiKO, Sesamum indicum entkaurene oxidase (XP011074464); SmKO, Salvia miltiorrhiza ent-kaurene oxidase (ALR74628); SmCYP88A52, S. miltiorrhiza cytochrome P450 (AJD25209); SiKAO, S. indicum ent-kaurenoic acid oxidase 2 (XP020548506); SmGA20ox2, S. miltiorrhiza gibberellin 20-oxidase 2 (ALR74634); SmGA20ox4, S. miltiorrhiza gibberellin 20-oxidase 4; SmGA3ox1, S. miltiorrhiza gibberellin 3-oxidase 1 (ALR74631); SIGA20ox1, Solanum lycopersicum gibberellin 20-oxidase 1, (ABS84235); TaGA3ox2, Triticum aestivum gibberellin 3-oxidase 2 (AAZ94378). Numbers on branches indicate the bootstrap percentage values calculated from 1000 bootstrap replicates

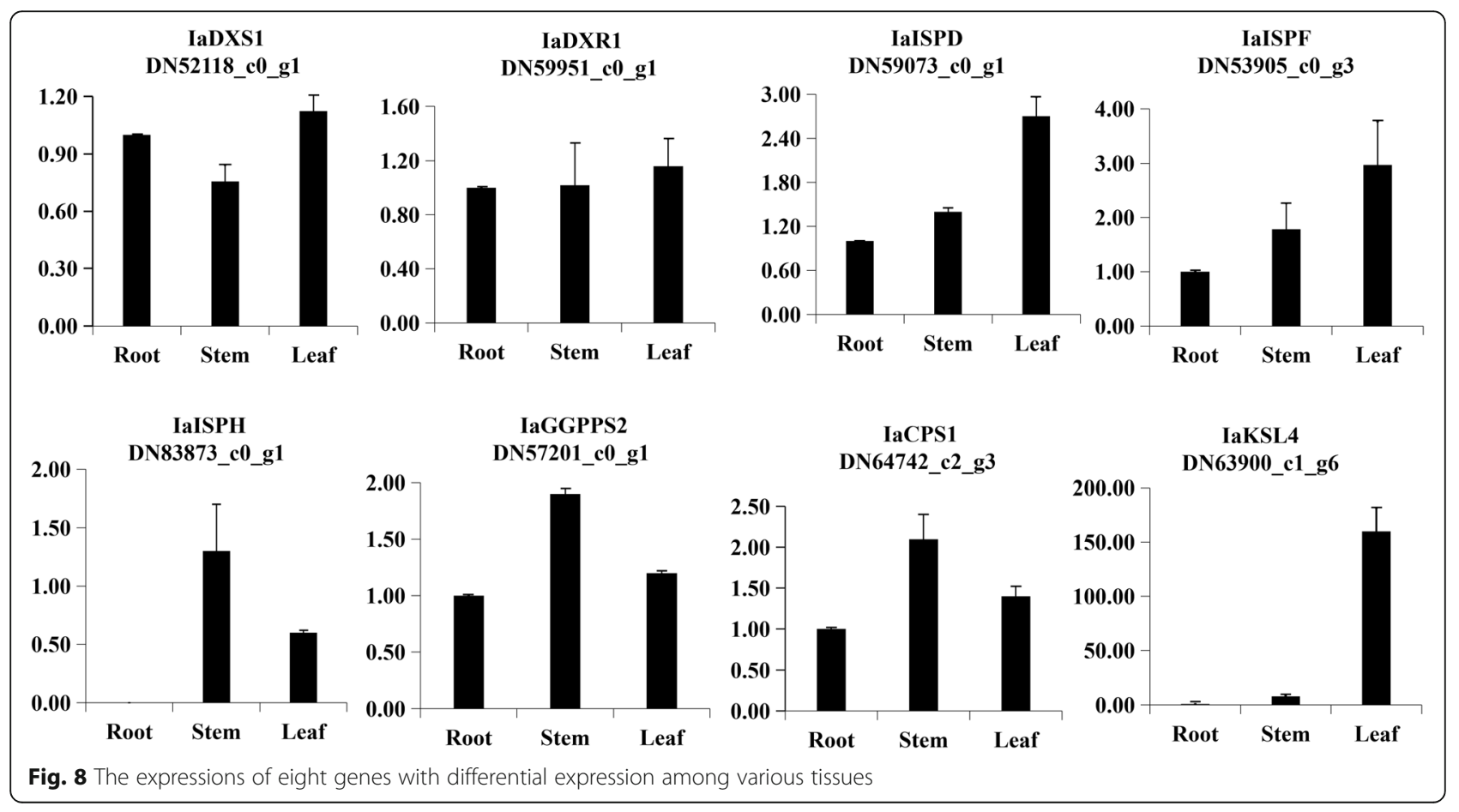


Functional annotation indicated that $64.37 \%$ (73695) of the transcripts could be assigned to at least one putative function from one of the COG, KEGG, Pfam, Swiss-Prot, or NR databases. This indicates the good de novo assembly of the I. amethystoides transcriptomic data. In its relative species Isodon reubescens, Su et al. [6] generated a dataset of 44,626 unigenes from leaf transcriptome, and $57.54 \%$ (25677) were annotated in one of the databases. In this study, we obtained more unigenes (approximately three folds) in I. amethystoides by comparative analysis of root, stem and leaves, which provide more abundant genetic information for understanding the molecular mechanism of Wangzoazins biosynthesis.

On the basis of the large-scale transcriptome data, identification of candidate genes of transcription factors (TFs) or those encoding key enzymes is essential for elucidating the biosynthesis of tetracyclic diterpenoids in $I$. amethystoides. TFs, which generally occur as gene families, play key roles in regulating the expression of genes by specifically binding to the cis-regulatory elements in the promoter regions of target genes [35]. Approximately $2 \%$ (1373) of the assembled transcripts were TFs, which could be classified into 55 TF families, including the C2H2, bZIP, and bHLH families. The roles of TFs in regulating diterpenoids have been widely studied in crops [36, 37]. For the Lamiaceae family, bZIP and bHLH genes have been found to be involved in the regulation of tanshinone biosynthesis [38, 39]. The evidence suggest that functional characterization of TFs has the potential to increase tetracyclic diterpenoid production. However, there is no reports focusing on the role of TFs in regulating diterpenoid biosynthesis in Isodon species. Thus, the candidate TFs provide a large gene pool to mine important regulatory genes for Wangzaozins production.

For the biosynthesis of Wangzoazins, 29 transcripts, including 14 in the MEP pathway and 15 in the tetracyclic diterpenoid biosynthesis were identified in this study. ISPD, $I S P F$, and ISPH have been found in our RNA-seq dataset as a single copy, consistent with the conclusion in Stevia rebaudiana [16]. All the three transcripts had a 2 to 3-fold higher expression in leaf than root. Four DXS and two DXR genes had constitutive expressions or similar expression among three tissues, which implies that these genes may play roles both in primary and diterpenoid metabolisms. The GGPPS family has been reported to be encoded by two to 12 members in plants [40]. Four IaGGPPS candidate genes were found in the present study, of which IaGGPPS1 (DN49479_c0_g1) showed higher expressions in above ground parts compared to the root.

Five CPS transcripts were screened out from the RNA-seq dataset, with similar expression among the three examined tissues, or preferential expression in leaf, stem, or root. From these CPS transcripts, we identified one (IaCPS3, DN34471_c0_g1) containing the complete open reading frame. Phylogenetic analysis revealed that it shares a high amino acid identity with $I$. rubescens IrCPS3. IrCPS3 was expressed five-fold higher in stem than root and leaf [19]. Other CPS transcripts share a high homology with known CPS genes from other plant species. Particularly, IaCPS1 was placed in the same clade with $I$. eriocalyx IeCPS2. IeCPS2 has been shown to specifically involved in the biosynthesis of pharmacologically active Isodon diterpenoids rather than gibberellin [18], implying a potential role of IaCPS1 in the biosynthesis of Wangzaozins.

The ent-kaurene synthase (EC 4.2.3.19) is an enzyme that catalyzes the formation of ent-kaurene from ent-copalyl diphosphate by releasing diphosphate. From the RNA-seq dataset, we identified four kaurene synthase-like genes in I. amethystoides. IaKSL2 and IaKSL4 are closely related to IrKSL5 and IrKSL4 respectively. In I. rubescens, IrKSL5 and IrKSL4 are involved in the generation of ent-kaurene [19]. It suggests that IaKSL2 and IaKSL4 may be the potential genes to react with ent-CPP in $I$. amethystoides.

The functional diversification of diterpene syntheses has been discovered in Salvia miltiorrhiza [41] and I. rubescens [19]. Therefore, the candidate genes identified in this study will make an important contribution to molecular studies on the biosynthesis of tetracyclic diterpenoid biosynthesis in I. amethystoides.

Gibberellins are diterpenoids that are derived biosynthetically from ent-kaurene, as some pharmacological diterpenoids $[18,19,27]$. In this study, the expressions of 9 transcripts associated with gibberellin biosynthesis were analyzed using the transcriptomic data. Except for IaGA3ox1 being expressed similarly among the three tissues, all other genes showed differential expression patterns. This infers the possible difference in GA production among diverse tissues of I. amethystoides.

\section{Conclusion}

We generated a comprehensive transcriptome assembly harboring genetic information for mass sequence data of I. amethystoides. These sequence data allowed us to identify and characterize the molecular functions of TFs and candidate transcripts associated with tetracyclic diterpenoid metabolic pathways, which provide insight into the biosynthesis of bioactive compounds in $I$. amethystoides. Particularly, twenty nine transcripts encoding key enzymes in tetracyclic diterpenoid biosynthesis were identified. The higher expressions of IaISPD, IaISPE and IaISPF (MEP pathway), and IaCPS (such as IaCPS1) and IaKSL (such as IaKSL2 and IaKSL4) candidate genes in leaves, may contribute to the high accumulation of Wangzaozins in I. amethystoides leaves. Intensive biochemical, enzymatic, physiological, and 
molecular studies on these candidate transcripts will be necessary to better understand the underlying regulatory mechanisms. Moreover, our study records the first genomics resource for I. amethystoides and lays the foundations for further improvements in this medicinal crop through genetic, genomic, and engineering methods.

\section{Methods}

\section{Plant materials}

One-year-old Isodon amethystoides (Ben-th) Cy Wu et Hsuan plants, identified by Prof. Jianping Xue (Huaibei Normal University), were collected from the Experimental Farm of Huaibei Normal University, Huaibei City, Anhui Province, P.R. China $\left(33^{\circ} 16^{\prime} \mathrm{N}, 116^{\circ} 23^{\prime} \mathrm{E}\right.$, altitude, $\left.340 \mathrm{~m}\right)$, in May 2015. Root, stem, and leaf samples were collected separately from randomly healthy plant individuals.

\section{Extraction and estimation of Wangzaozins}

Each tissue was pooled from three plants, air-dried, and ground into a fine powder. One gram of powder was weighed into $20 \mathrm{~mL}$ of ethanol for ultrasonication extraction at room temperature for $1 \mathrm{~h}$. The extract was collected by filtering and then dried via rotary evaporation. The yielded pellet was dissolved in methanol to an appropriate concentration.

Standard references of Wangzaozins (Wangzaozin A, glaucocalyxin A, and glaucocalyxin B) were purchased from Chengdu Purechem-standard Co., Ltd. (Chengdu, China). Each standard reference was dissolved in methanol to yield a $0.1 \mathrm{mg} \cdot \mathrm{mL}^{-1}$ solution.

An Agilent Technologies 1260 Infinity Series HPLC system equipped with a Thermo-C18 column $(250 \mathrm{~mm} \times$ $4.6 \mathrm{~mm}, 5.0 \mu \mathrm{m}$ ) was used for liquid chromatographic separations. The mobile phase consisted of $0.1 \%$ acetic acid in water (solvent A) and methanol (solvent B). The gradient elution was as follows: $0-6$ min, linear from 40 to $45 \% \mathrm{~B} ; 6-35 \mathrm{~min}$, held at $45 \% \mathrm{~B} ; 35-45 \mathrm{~min}$, linear from 45 to $95 \% \mathrm{~B}$. The flow rate was $1.0 \mathrm{~mL} / \mathrm{min}$. The injection volume was $10 \mu \mathrm{L}$. The column temperature was set at $30{ }^{\circ} \mathrm{C}$ and the sampler was set at $4{ }^{\circ} \mathrm{C}$.

The contents of Wangzaozin A, glaucocalyxin A, and glaucocalyxin $B$ in each tissue were quantified by calculating the peak area compared with those of the respective standards by linear regression. The content was presented as an average percentage of dry weight (DWT) from three replicates.

\section{RNA extraction and transcriptome sequencing}

All plant samples were separately cut into small pieces, and $2.0 \mathrm{~g}$ (fresh weight) of each tissue pooled from three plants was used for RNA isolation. The experiments contained three biological replications, and thus nine plants were used for each tissue. Total RNA extraction and RNA integrity evaluation were performed according to a previously published protocol [42]. RNA sequencing libraries with an average insert length of 250 bp were sent Genepioneer Technologies Corporation (Nanjing, China) for sequencing on Illumina HiSeq4000 platform (Illumina Inc., USA).

\section{Data filtering and de novo assembly}

The raw data of nine RNA sequencing reads were trimmed to remove the adaptor sequences, duplicated sequences, ambiguous reads ('N'), and low-quality reads. The yielded clean reads were then used for sequence assembly using the Trinity program with default parameters [43]. Contigs were generated by combining the clean reads with a certain overlap and then clustered using TGICL software [44] to yield unigenes. Non-redundant unigenes were acquired by removing the redundancies $(-140,-\mathrm{c} 10$, and $-\mathrm{v} 20)$.

\section{Functional annotation and classification}

The unique sequences were annotated against public databases, including the NR (http://www.ncbi.nlm.nih.gov/), Swiss-Prot (http://www.expasy.ch/sprot), Clusters of Orthologous Groups (COG) (http://www.ncbi.nlm.nih.gov/COG/), and Pfam (http://pfam.xfam.org/) databases, using BLASTX (http://blast.ncbi.nlm.nih.gov/Blast.cgi) with an $\mathrm{E}$ value of $1 \mathrm{e}^{-5}$ [45]. GO analysis was performed to obtain the Gene Ontology (GO) functional classifications [46]. KEGG classification maps were drawn based on the retrieved Kyoto Encyclopedia of Genes and Genomes (KEGG) Orthology (KO) information. The transcription factors (TFs) in the I. amethystoides transcriptome were identified by searching the plant transcription factor database PlantTFDB 4.0 with all assembled unigenes [47].

\section{Differentially expressed genes (DEGs)}

The fragments per kb per million reads (FPKM) method was employed to quantify the transcript abundances [26]. The thresholds for the significance test were set as FDR value $\leq 0.01$ and the absolute value of $\log 2$ ratio $\geq 1$. Each set of differentially expressed unigenes was analyzed by hypergeometric Fisher exact test $(P<0.01)$ and Benjamini correction $(\mathrm{FDR}<0.05)$ to construct the KEGG pathways.

\section{Verification of gene expression using qRT-PCR}

Eight DEGs related to Wangzaozins biosynthesis were selected for quantitative real-time PCR analysis. The primer sequences used are listed in Additional file 15: Table S1. qRT-PCR was performed using the ABI7500 fast Real-Time PCR system (Applied Biosystems, USA) with a SYBR ${ }^{\circ}$ Premix ExTaq ${ }^{\text {Tw }}$ Kit (Takara, Dalian, China). The Actin gene was used for normalization of expression level. Three biological replicates and three technical 
repeats were contained for each gene and sample. The $2^{-\Delta \Delta C T}$ method was used to calculate the relative gene expression [48].

\section{Additional files}

Additional file 1: Table S2. Overview of transcriptome sequencing and de novo assembly. (DOC $18 \mathrm{~kb}$ )

Additional file 2: Figure S1. Overview of transcriptome assembly showing size distribution. (TIF $714 \mathrm{~kb}$ )

Additional file 3: Table S3. Correlation indices between different samples. (XLSX $9 \mathrm{~kb}$ )

Additional file 4: Figure S2. Correlation indices between different samples (PDF $4 \mathrm{~kb}$ )

Additional file 5: Table S4. Summary of functional annotation of contigs from BLAST searches against public databases. (DOCX $12 \mathrm{~kb}$ )

Additional file 6: Figure S3. Homologous species of Isodon amethystoides transcriptomes. (TIF $110 \mathrm{~kb}$ )

Additional file 7: Table S5. Transcription factors identified in this study. (XLSX $116 \mathrm{~kb}$ )

Additional file 8: Figure S4. Distribution of transcription factor families. (TIF $1288 \mathrm{~kb}$ )

Additional file 9: Figure S5. Frequencies and mean expression levels of transcripts matching GO terms. The percentage of transcripts matching GO terms is shown for each category. (PNG $139 \mathrm{~kb}$ )

Additional file 10: Table S6. GO enrichment of unigenes. (XLS 8009 kb)

Additional file 11: Table S7. Unigenes for KEGG analysis. (XLSX 667 kb)

Additional file 12: Table S8. DEGs between root, stem and leaf. (XLSX 2009 kb)

Additional file 13: Table S9. KEGG enrichment analysis list. (XLS 73 kb)

Additional file 14: Table S10. Summary of transcripts in Isodon amethystoides encoding enzymes involved in the tetracyclic terpenoid biosynthesis. (DOCX $22 \mathrm{~kb}$ )

Additional file 15: Table S1. The primers used for qRT-PCR. (DOC $34 \mathrm{~kb}$ )

\section{Abbreviations}

CPS: ent-copalyl diphosphate synthase; DEGs: Differentially expressed genes; DMAPP: Dimethylallyl diphosphate; DWT: Dry weight; DXR: 1-deoxy-dxylulose-5-phosphate reductoisomerase; FPKM: Fragments per kilobase per million reads; GGPP: Geranyl geranyl-PP; IPP: Isopentenyl diphosphate; ISPD: 2-C-methyl-d-erythritol 4-phosphate cytidylyltransferase; ISPF: 2CMethyl-D-erythritol 2,4-cyclodiphosphate synthase; ISPG: 4-hydroxy-3methylbut-2-en-1-yl diphosphate synthase; ISPH: 1-hydroxy-2-methyl-2-(E)butenyl 4-diphosphate reductase; KAO: ent-kaurenoic acid oxidase; KOS: entkaurene oxidase; KSL: ent-kaurene synthase-like; MEP: Methylerythritol phosphate; qRT-PCR: Quantitative reverse transcription PCR

\section{Acknowledgements}

The authors would like to thank Prof. Li Li (Cornell University) for valuable comments and suggestions, and Dr. Tara Fish (USDA-ARS, Robert W. Holley Center for Agriculture and Health) for English polishing.

\section{Funding}

This work was financially supported by the National Natural Science Foundation of China $(31501368,81573518)$, the Natural Science Foundation of China (1608085MC52), and the Project of Natural Science Research of Universities in Anhui Province, China (KJ2016B016; KJ2016A644; KJ2017A846). The Funding bodies were not involved in the design of the study and collection, analysis, and interpretation of data and in writing the manuscript.

\section{Availability of data and materials}

All relevant data are available within the manuscript. The RNA-Seq reads used for this study were deposited at the National Center for Biotechnology
Information Short Read Archive (http://www.ncbi.nlm.nih.gov/sra/) under the accession number SRP 117767.

\section{Authors' contributions}

YD and JX conceived and designed the experiments. YD and JX collected the materials. FZ, YD, MS, WZ, CJ, WS and AZ performed the experiments. FL, YD, JT, ML and JX compiled and interpreted the data. YD, FZ, and JX wrote the manuscript. All authors reviewed and approved the manuscript.

Ethics approval and consent to participate

Not applicable.

\section{Consent for publication}

Not applicable.

\section{Competing interests}

The authors declare that they have no competing interests.

\section{Publisher's Note}

Springer Nature remains neutral with regard to jurisdictional claims in published maps and institutional affiliations.

\section{Author details}

${ }^{1}$ Key Laboratory of Resource Plant Biology of Anhui Province, College of Life Sciences, Huaibei Normal University, Huaibei City, China. ${ }^{2}$ Genepioneer Biotechnologies Co. Ltd, Nanjing City, China.

Received: 5 February 2018 Accepted: 26 October 2018 Published online: 08 November 2018

\section{References}

1. Yu XQ, Maki M, Drew BT, Paton AJ, Li HW, Zhao JL, Conran JG, Li J. Phylogeny and historical biogeography of Isodon (Lamiaceae): rapid radiation in south-West China and Miocene overland dispersal into Africa. Mol Phylogen Evol. 2014;77:183-94.

2. Yang J, Wang WG, Wu HY, Liu M, Jiang HY, Du X, Li Y, Pu JX, Sun HD. Entkaurane diterpenoids from Isodon phyllostachys. Tetrahed Letts. 2016;58:349-51.

3. Matsumoto T, Nakamura S, Kojima N, Hasei T, Yamashita M, Watanabe T, Matsuda $\mathrm{H}$. Antimutagenic activity of ent-kaurane diterpenoids from the aerial parts of Isodon japonicus. Tetrahed Letts. 2013;58:3574-8.

4. Lu Y, Chen B, Song JH, Zhen T, Wang BY, Li X, Liu P, Yang X, Zhang QL, Xi XD, Chen SD, Zuo JP, Chen Z, Chen SJ. Eriocalyxin B ameliorates experimental autoimmune encephalomyelitis by suppressing Th1 and Th17 cells. Proc Natl Acad Sci U S A. 2013;110:2258-63.

5. Yang J, Wang WG, Wu HY, Du X, Li XN, Li Y, Pu JX, Sun HD. Bioactive enmein-type ent-kaurane diterpenoids from Isodon phyllostachys. J Nat Prod. 2016;79:132-40.

6. Su X, Li Q, Chen S, Dong C, Hu Y, Yin L, Yang JF. Analysis of the transcriptome of Isodon rubescens and key enzymes involved in terpenoid biosynthesis. Biotech Biotechnol Equip. 2016;30:592-601.

7. Chen Z, Li Y, Zhou J, Cao L, Li Y. Immunopharmacological effect of Rabdosia amethystoides (Benth.) Hara in mice. Chin Pharm J. 2006:41:908-10.

8. Zhang $\mathrm{XH}$. Clinical application of antimicrobial effect of medicinal herb Isodon amethystoides (ben-th) cy Wu et Hsuan. Coal Med. 1982:4:13-5.

9. Li GG, Wang YL, Xu ZP, Zhang PL, Zhao W. Studies on the chemical constituents of Isodon amethystoides (Benth.) cy Wu et Hsuan. Acta Pharm Sin. 1981;9:667-71

10. Wang XR, Wang ZQ, Shi PC, Zhou BN. A novel diterpenoid of Isodon amethystoides (ben-th) cy Wu et Hsuan-hydroglaucocalyxin a. Anhui Med. 1982;2:50-3

11. Tang L, Jin X, Hu X, Hu X, Liu Z, Yu L. Glaucocalyxin a inhibits the growth of liver cancer focus and SMMC-7721 cells. Oncol Lett. 2016;11:1173e1178.

12. Gao LW, Zhang J, Yang WH, Wang B, Wang JW. Glaucocalyxin a induces apoptosis in human leukemia $\mathrm{HL}-60$ cells through mitochondria-mediated death pathway. Toxicol in Vitro. 2011:25:51e63.

13. Li M, Jiang XG, Gu ZL, Zhang ZB. Glaucocalyxin a activates Fasl and induces apoptosis through activation of the Jnk pathway in human breast cancer cells. Asian Pac J Cancer Prev. 2013;14:5805e5810.

14. Kim BW, Koppula S, Hong SS, Jeon SB, Kwon JH, Hwang BY, Park EJ, Choi DK. Regulation of microglia activity by glaucocalyxin-a: attenuation of 
lipopolysaccharide-stimulated neuroinflammation through NF-kb and p38 MAPK signaling pathways. PLoS One. 2013;8:e55792.

15. Xiang Z, Wu X, Liu X, Jin Y. Glaucocalyxin a: a review. Nat Prod Res. 2014;28: 2221-36.

16. Kim MJ, Jin J, Zheng J, Wong L, Chua NH, Jang IC. Comparative transcriptomics unravel biochemical specialization of leaf tissues of Stevia for diterpenoid production. Plant Physiol. 2015;4:2462-80.

17. Yang L, Yang C, Li C, Zhao Q, Liu L, Fang X, Chen XY. Recent advances in biosynthesis of bioactive compounds in traditional Chinese medicinal plants. Sci Bull (Beijing). 2016;61:3-17.

18. Li JL, Chen QQ, Jin QP, Gao J, Zhao PJ, Lu S, Zeng Y. leCPS2 is potentially involved in the biosynthesis of pharmacologically active Isodon diterpenoids rather than gibberellin. Phytochemistry. 2012;76:32-9.

19. Jin BL, Cui GH, Guo J, Tang JF, Duan LX, Lin HX, Shen Y, Chen T, Zhang HB, Huang LQ. Functional diversification of kaurene synthase-like genes in Isodon rubescens. Plant Physiol. 2017;174:943-55.

20. Pelot KA, Hagelthorn DM, Addison JB, Zerbe P. Biosynthesis of the oxygenated diterpene nezukol in the medicinal plant Isodon rubescens is catalyzed by a pair of diterpene synthases. PLoS One. 2017:4:e0176507.

21. Zerbe P, Hamberger B, Yuen MM, Chiang A, Sandhu HK, Madilao LL, Nguyen A, Hamberger B, Bach SS, Bohlmann J. Gene discovery of modular diterpene metabolism in non-model systems. Plant Physiol. 2013;2:1073-91.

22. Nakano Y, Yamaguchi M, Endo H, Rejab NA, Ohtani M. NAC-MYB-based transcriptional regulation of secondary cell wall biosynthesis in land plants. Front Plant Sci. 2015;6:288.

23. Chen K, Liu H, Lou Q, Liu Y. Ectopic expression of the grape hyacinth (Muscari armeniacum) R2R3-MYB transcription factor gene, MaAN2, induces anthocyanin accumulation in tobacco. Front Plant Sci. 2017:8:965.

24. Yang L, Ding $G$, Lin $H$, Cheng $H$, Kong Y, Wei Y, Fang X, Liu PY, Wang L, Chen XY, Yang CQ. Transcriptome analysis of medicinal plant Salvia miltiorrhiza and identification of genes related to tanshinone biosynthesis. PLoS One. 2013;8:e80464.

25. Gao W, Sun HX, Xiao H, Cui G, Hillwig ML, Jackson A, Wang X, Shen Y, Zhao $\mathrm{N}$, Zhang L, Wang XJ, Peters RJ, Huang L. Combining metabolomics and transcriptomics to characterize tanshinone biosynthesis in Salvia miltiorrhiza. BMC Genomics. 2014;15:73.

26. Wang LK, Feng ZX, Wang X, Wang XW, Zhang XG. DEGseq: an R package for identifying differentially expressed genes from RNA-seq data. Bioinformatics. 2010;26:136-8.

27. Zi J, Mafu S, Peters RJ. To gibberellins and beyond! Surveying the evolution of (di)terpenoid metabolism. Annu Rev Plant Biol. 2014;1:259-86.

28. Alvarez MA. Plant biotechnology for health: from secondary metabolites to molecular farming, vol. 8. Cham: Springer International Publishing; 2014. p. 39-48.

29. Wang Z, Gerstein M, Snyder M. RNA-seq: a revolutionary tool for transcriptomics. Nat Rev Genet. 2009;10:57-63.

30. Ponniah SK, Thimmapuram J, Bhide K, Kalavacharla V, Manoharan M. Comparative analysis of the root transcriptomes of cultivated sweetpotato (Ipomoea batatas [L.] Lam) and its wild ancestor (Ipomoea trifida [Kunth] G. Don). BMC Plant Biol. 2017;17:9.

31. Liu Y, Wang Y, Guo FX, Zhan L, Mohr T, Cheng P, Huo N, Gu RH, Pei DN, Sun JQ, Li T, Long CL, Huang LQ, Gu YQ. Deep sequencing and transcriptome analyses to identify genes involved in secoiridoid biosynthesis in the Tibetan medicinal plant Swertia mussotii. Sci Rep. 2017;7:43108.

32. Garg A, Agrawal L, Misra RC, Sharma S, Ghosh S. Andrographis paniculato transcriptome provides molecular insights into tissue-specific accumulation of medicinal diterpenes. BMC Genomics. 2015;6:659.

33. Cherukupalli N, Divate M, Mittapelli SR, Khareedu VR, Vudem DR. De novo assembly of leaf transcriptome in the medicinal plant Andrographis paniculata. Front Plant Sci. 2016;7:1203.

34. Zhang L, Jia H, Yin Y, Wu G, Xia H, Wang X, Fu CH, Li MT. Transcriptome analysis of leaf tissue of Raphanus sativus by RNA sequencing. PLoS One. 2013:8:e80350

35. Woldemariam MG, Dinh ST, Oh Y, Gaquerel E, Baldwin IT, Galis I. NaMYC2 transcription factor regulates a subset of plant defense responses in Nicotiana attenuata. BMC Plant Biol. 2013;1:73.

36. Miyamoto K, Matsumoto T, Okada A, Komiyama K, Chujo T, Yoshikawa H, Okada K. Identification of target genes of the bZIP transcription factor ostgap1, whose overexpression causes elicitor-induced hyperaccumulation of diterpenoid phytoalexins in rice cells. PLoS One. 2014:8:e105823.
37. Yamamura C, Mizutani E, Okada K, Nakagawa H, Fukushima S, Tanaka A, Maeda S, Kamakura T, Yamane H, Takatsuji H, Mori M. Diterpenoid phytoalexin factor, a bHLH transcription factor, plays a central role in the biosynthesis of diterpenoid phytoalexins in rice. Plant J. 2016;6:1100-13.

38. Zhang $Y, X u Z$, Ji A, Luo H, Song J. Genomic survey of bZIP transcription factor genes related to tanshinone biosynthesis in Salvia miltiorrhiza. Acta Pharm Sin B. 2018;2:295-305.

39. Zhang X, Luo H, Xu Z, Zhu Y, Ji A, Song J, Chen SL. Genome-wide characterisation and analysis of bHLH transcription factors related to tanshinone biosynthesis in Salvia miltiorrhiza. PLoS One. 2015;5:11244.

40. Beck G, Coman D, Herren E, Ruizsola MA, Gruissem W. Characterization of the GGPP synthase gene family in Arabidopsis thaliana. Plant Mol Biol. 2013; 4:393-416.

41. Cui G, Duan L, Jin B, Qian J, Xue Z, Shen G, Snyder JH, Song J, Chen S, Huang L, Peters RJ, Qi X. Functional divergence of diterpene syntheses in the medicinal plant Salvia miltiorrhiza. Plant Physiol. 2015;3:1607-18.

42. Zhang XH, Berkowitz O, Silva JATD, Zhang MH, Ma GH, Whelan J, Duan J. RNA-seq analysis identifies key genes associated with haustorial development in the root hemiparasite Santalum album. Front Plant Sci. 2015;6:661.

43. Haas BJ, Papanicolaou A, Yassour M, Grabherr M, Blood PD, Bowden J, Couger MB, Eccles D, Li B, Lieber M, MacManes MD, Ott M, Orvis J, Pochet N, Strozzi F, Weeks N, Westerman R, William T, Dewey CN, Henschel R, LeDuc RD, Friedman N, Regev A. De novo transcript sequence reconstruction from RNA-Seq: reference generation and analysis with trinity. Nat Protoc. 2013:8:1494-512.

44. Pertea G, Huang X, Liang F, Antonescu V, Sultana R, Karamycheva S, Lee Y, White J, Cheung F, Parvizi B, Tsai J, Quachenbush J. TIGR gene indices clustering tools (TGICL): a software system for fast clustering of large EST datasets. Bioinformatics. 2003;19:651-2.

45. Ye BH, Zhang YB, Shu JP, Wu H, Wang HJ. RNA-sequencing analysis of fungi-induced transcripts from the bamboo wireworm Melanotus cribricollis (Coleoptera: Elateridae) larvae. PLoS One. 2018:13(1):e0191187.

46. Ye J, Fang L, Zheng H, Zhang Y, Chen J, Zhang Z, Wang J, Li ST, Li RQ, Bolund L, Wang J. WEGO: a web tool for plotting GO annotations. Nucl Acids Res. 2006;34:293-7.

47. Jin JP, Tian F, Yang DC, Meng YQ, Kong L, Luo JC, Gao G. PlantTFDB 4.0: toward a central hub for transcription factors and regulatory interactions in plants. Nucl Acids Res. 2017:45:1040-5.

48. Pfaffl MW. A new mathematical model for relative quantification in real-time RT-PCR. Nucl Acids Res. 2001;29(9):e45.

\section{Ready to submit your research? Choose BMC and benefit from:}

- fast, convenient online submission

- thorough peer review by experienced researchers in your field

- rapid publication on acceptance

- support for research data, including large and complex data types

- gold Open Access which fosters wider collaboration and increased citations

- maximum visibility for your research: over $100 \mathrm{M}$ website views per year

At $\mathrm{BMC}$, research is always in progress.

Learn more biomedcentral.com/submissions 